\title{
Automatic nematode detection in cod fillets (Gadus Morhua L.) by hyperspectral imaging
}

\author{
Agnar Holten Sivertsen ${ }^{\mathrm{a}, *}$, Karsten Heia ${ }^{\mathrm{a}}$, Kristian Hindberg ${ }^{\mathrm{b}}$, Fred \\ Godtliebsen $^{\mathrm{b}}$ \\ ${ }^{a}$ Nofima AS, Pb 6122, 9291 Tromsø,Norway \\ ${ }^{b}$ University of Tromsø, Tromsø, Norway
}

\begin{abstract}
Detection of objects embedded in tissue, using visible light, is difficult due to light scattering. The optical properties of the surrounding tissue will influence the spectral characteristics of the light interacting with the object, and the spectral signature observed from the object will be directly affected. A method for calibrating the spectral signature of small objects, embedded in translucent material, by the estimated local background spectrum is presented. The method is evaluated under industrial conditions in a new hyperspectral imaging system for automatic detection of nematodes in cod fillets. The system operates at a conveyor belt speed of $400 \mathrm{~mm} / \mathrm{second}$ which meets the industrial required speed of assessing one fillet per second. The local calibration method reduces the number of spectra needed to be classified by $89.6 \%$. For one or more false alarms in $60 \%$ of the fillets sampled after the trimming station, the Gaussian maximum likelihood classifier

\footnotetext{
*Corresponding author

Email addresses: agnarhs@nofima.no (Agnar Holten Sivertsen),
} karstenh@nofima.no (Karsten Heia), kristian.hindberg@uit.no (Kristian Hindberg), fred.godtliebsen@uit.no (Fred Godtliebsen)
\end{abstract}


detects $70.8 \%$ and $60.3 \%$ of the dark and pale nematodes, respectively. This is better than what is previously reported using a higher resolution instrument on a slow moving conveyor belt, and comparable or better to what is reported for manual inspection under industrial conditions.

Keywords: hyperspectral imaging, imaging spectroscopy, industrial fish fillet inspection, image processing, local calibration

\section{Introduction}

Hyperspectral imaging (HSI), also known as imaging spectroscopy, is an emerging analytical tool integrating imaging and spectroscopy to attain a full spectral profile of each point in a scene being imaged. HSI was initially developed for remote sensing applications, but has increasingly been adopted in food control applications. Most applications, 22 out of 30 research papers since 2004 (Gowen et al., 2007), have utilized HSI in reflectance mode. Recently, HSI has been applied to problems requiring other measurement modes such as transmission for detection of nematodes in cod fillets (Sivertsen et al., 2011a) and interactance for estimating freshness of cod fillets (Sivertsen et al., 2011b), water content of cliff fish (Wold et al., 2006), ice fraction (Ottestad et al., 2009) and fat content (Segtnan et al., 2009) in salmon fillets.

The two main types of parasitic nematodes infecting Atlantic cod ( Gadus morhua) are Anisakis simplex and Pseudoterranova decipiens. A. simplex is more abundant in offshore fish, whereas $P$. decipiens is more likely to be found in inshore fish (Marcogliese, 2002). The two nematode species differ in size and color, where P. decipiens is often both darker and larger than A. simplex. Consuming nematode infected fish has traditionally not been considered a 
health risk as long as the nematode is killed by adequate cooking, freezing or frozen storage (Wharton and Aalders, 2002). Nematodes have mainly been considered a cosmetic problem, which can have a significant impact on fish consumption (Fischler, 2002). As undercooked seafood increases in popularity the risk of nematodes infecting humans increases. Lately, the potential for A. Simplex to induce hypersensitive reactions in humans, even after it has been killed by cooking or freezing, has received increased attention (Werner et al., 2011).

Today every single fillet is inspected by transillumination on candling tables (Hafsteinsson and Rizvi, 1987), and nematodes are removed manually. This is referred to as trimming, and is an expensive operation previously reported to account for half of the production cost for Pacific cod from the Bering Sea and the Gulf of Alaska (Bublitz and Choudhury, 1992). The fillet trimming is a bottleneck of the current fillet processing industry and often performed in room temperature, around $20^{\circ} \mathrm{C}$, with an increased risk of bacterial and enzymatic degradation. The manual detection efficiency for $P$. decipiens is reported as $68 \%$ under ideal conditions, as low as $50 \%$ under industrial conditions (Hafsteinsson and Rizvi, 1987) and only $25 \%$ for fillets with skin (Hauksson, 1991). Others have reported detection rates in the range 33 - $93 \%$ (Varga and Anderson, 1971), with an average of $68 \%$ (Bublitz and Choudhury, 1992). The absorbance characteristics of nematodes differ from cod muscle in the region 370-600 nm (Stormo et al., 2007, 2004; Petursson, 1991). In this region scattering of light is prominent in cod muscle and light interacting with the nematode is mixed with light from the surrounding tissue. This is why nematodes embedded deeper than 4-6 
mm are not detected by manual inspection (Bublitz and Choudhury, 1992;

Hafsteinsson et al., 1989).

Automatic nematode detection has been a prioritized research area for the cod fillet industry, where a series of different methods have been evaluated (see Sivertsen et al. (2011a); Heia et al. (2007) for a list of references). These methods have been evaluated at laboratory scale and on small fillet segments, and none have so far made it to an industrial application. Recently a system capable of automatic detection of nematodes in full size cod fillets was presented (Sivertsen et al., 2011a). The system utilized HSI in transmission mode, and was operating at a belt speed of $25 \mathrm{~mm} / \mathrm{second}$. The system was evaluated on industrially processed fillets and the performance was comparable to manual detection on candling tables. However, the limitations with the system are the slow speed and that it may not be used on fillets with skin.

Several methods for preprocessing optical spectra to reduce the effect of scattering have been developed. Examples are the second derivative calculated using the Savitzky-Golay second order smoothing filter (Savitzky and Golay, 1964), standard normal variate (SNV) (Barnes et al., 1989) and multiplicative scatter correction (Geladi et al., 1985). These are all common methods applied in spectroscopy and work well when one can assume homogeneous samples were the absorbance and scattering properties are constant along the optical path. This assumption does not apply for HSI of fish fillets, where the sample thickness, geometry and optical path length varies across the sample. In addition the light often propagates through muscle layers or regions with different optical properties, making it a non-trivial problem 
to separate the absorbance and scattering effect in the recorded HSI spectra. The recorded spectra from two similar nematodes embedded in different muscle regions can be different, even though the absorbance characteristic of the two nematodes are identical. This will result in large spectral variations, and hence a difficult classification problem.

The main objective of this work was to build a HSI setup for automatic detection of nematodes in cod fillets, operating at the industrial speed of 400 $\mathrm{mm} /$ second with a performance comparable to what is achieved with manual inspection on candling tables.

\section{Materials and methods}

All the image processing methods and algorithms explained in the following were implemented in IDL (Exelis, Inc.).

\subsection{Industrial test}

The test was performed at a fish processing plant in northern Norway during the period 2-3 March 2010. The inspection machine was installed at the plant two weeks earlier and 43 fillets were sampled after the skinning machine and inspected in order to adjust the instrumentation and train the classifier. These fillets, referred to as the training set, were only inspected from the fillet side and no depth registrations of the nematodes were done.

During the main test, 127 fillets were sampled prior to the trimming stations and 20 fillets were sampled after the trimming stations. These fillets are referred to as the test set. The fillets were sampled in batches of 10 and sent through the imaging machine. Each fillet was then manually inspected on a candling table, from both sides, by a team of two trained persons. To 
speed up the manual inspection, two different teams were used, each team inspecting half of the fillets. All spots that resembled a nematode infection were sliced with a knife and further inspected. A custom made computer program was used to manually pin-point each of the nematodes directly on to the image recorded of the fillet. Each nematode was classified as pale or dark, according to its white/yellow or red/brown color respectively. In addition each nematode was classified as a surface (0-2 mm), embedded (2$6 \mathrm{~mm}$ ) or deeply embedded nematode (deeper than $6 \mathrm{~mm}$ ). The deeply embedded nematodes were all found by inspecting the fillets from the skin side.

Nematodes found laying loose on top of the fillet, and which could not be seen in the image, were labeled with unknown position (UP).

\subsection{Hyperspectral interactance imaging system}

For the HSI system to meet the industrial speed requirements, several improvements were made to the hardware and measurement setup. These improvements and more details regarding the system are further explained in Sivertsen et al. (2011b). A detailed sketch of the main system components are shown in Fig. 1A, and a photograph of the inspection machine, with the front cover removed, is shown in Fig. 1B.

The camera in the spectrometer uses a charge coupled device (CCD) sensor with a full well capacity of 40000 electrons and 12 bit A/D converter. The sensor is equipped with anti-blooming gates (Janesick, 2001) and black clamping (Barron et al., 1995). The black clamping works by calculating a mean dark current value, from pixels around the CCD not exposed to light, and subtracting this from the sensor readout before the values are converted 
to digital numbers (DN).

A photo cell is positioned $200 \mathrm{~mm}$ in front of the measurement region, where a microcontroller reads the output from the photocell and trigger the spectrometer once a fillet is present on the conveyor belt.

Each of the spectra recorded by the HSI system represents light intensity from a spatial region of size $0.5 \mathrm{~mm} \times 1.0 \mathrm{~mm}$ in the region $400-1000 \mathrm{~nm}$ with a spectral resolution of approximately $10 \mathrm{~nm}$. The wavelength range 448-752 nm was used for all the analysis in this work, and this overlaps with the wavelength region previously reported to be well suited for discriminating nematodes from fish muscle (Stormo et al. (2007, 2004); Petursson (1991)).

\subsection{System calibration}

The spatial and spectral distribution across the field of view (FOV) is measured by imaging a $300 \mathrm{~mm}$ x $300 \mathrm{~mm}$ x $25 \mathrm{~mm}$ Teflon slab. This is done every time the system is initialized and stable, approximately 30 minutes after the system is powered up. A rectangular spatial region in the image of the Teflon target, approximately $200 \mathrm{~mm}$ x $40 \mathrm{~mm}$, is manually selected. This region is used to estimate the average spectral response across the field of view, $\hat{T}(s, \lambda)=(1 / N) \sum_{l^{*}} J\left(s^{*}, \lambda, l^{*}\right)$, where $\left(s^{*}, l^{*}\right)$ represent pixels inside the selected region, $J(s, \lambda, l)$ is the recorded interactance image of the Teflon target, $\lambda$ is the wavelength and $N$ is the number of lines in the selected region.

Each pixel, representing a spectrum from the corresponding region on the cod fillet being imaged, is then calibrated by

$$
I(s, \lambda, l)=\frac{J(s, \lambda, l)}{\hat{T}(s, \lambda)} .
$$




\subsection{Hyperspectral image model}

Due to the Poisson properties of the signal recorded from the CCD sensor (Benvenuto et al., 2008; Snyder et al., 1995), and neglecting the readout noise, the calibrated image can be approximated by

$$
I(s, \lambda, l) \sim \mathcal{N}(\bar{I}(s, \lambda, l), C(s, \lambda) \bar{I}(s, \lambda, l))
$$

where $\bar{I}(s, \lambda, l)$ is the expected intensity value, $C(s, \lambda)=\alpha / \hat{T}(s, \lambda), \alpha$ is the camera gain Janesick (2001) and $\mathcal{N}(\cdot)$ represent the normal distribution.

\subsection{Image segmentation}

All the pixels on the fillet are identified using three wavelengths $(500,646$ and $800 \mathrm{~nm}$ ), by the equation

$$
M(\mathbf{x})=\mathbf{1}_{\left(I\left(s, \lambda_{646}, l\right)>3.5\right)} \mathbf{1}_{\left(I\left(s, \lambda_{800}, l\right)>1.5 I\left(s, \lambda_{500}, l\right)\right)},
$$

where $\mathbf{1}_{(\cdot)}$ is the indicator function (Folland, 1999) and $\mathbf{x}=(s, l) . \quad M(\mathbf{x})$ equals one for pixels representing areas on the fillet and zero outside the fillet area. The fillet is further segmented into its respective parts using the centreline as a reference (Sivertsen et al., 2009). The fillet is divided into three parts: loin, belly and tail, where the transition between tail and loin/belly is set to $55 \%$ of the fillet length. The loin part is separated from the belly by the centerline, and defined as the part with the highest average value of $I\left(s, \lambda_{525}, l\right)$ inside the loin and belly part, respectively.

\subsection{Local calibration filter}

When doing measurements in interactance or transmission mode, one can assume that the light interacting with a nematode near the fillet surface is 
similar to the light registered from an area next to the nematode. The purpose of the local calibration filter is to calibrate each spectrum with the local background spectrum and reduce spectral variation within the nematode class due to background variations, such as fillet color and scattering properties. For simplicity we will consider a single band image in the following. The local mean value is calculated as

$$
\hat{I}(\mathbf{x})=\frac{\sum_{(x, y) \in A} K(u) M(x, y) I(x, y)}{\sum_{(x, y) \in A} K(u) M(x, y)},
$$

where $u=\left(\sqrt{(x-s)^{2}+(y-l)^{2}}\right) / r_{1}, M(\mathbf{x})$ is defined in (3) and $A\left\{\mathbf{x} ; r_{1}\right\}=$ $\{(x, y):|u| \leq 1\}$ defines the local neighborhood for the pixel in position $\mathbf{x}=(s, l)$. The parameters $r_{1}, r_{2}$ and $r_{3}$ defines the size and position of the local neighborhood and local background for the local calibration filter as illustrated in Fig. 2. The kernel, $K$, is the 1D Epanechnikov kernel (Epanechnikov, 1969) $K_{e}(u)=c\left(1-u^{2}\right) \mathbf{1}_{|u| \leq 1}$, where $c$ is a normalizing constant. The local calibrated image is then defined as

$$
L(\mathbf{x})=\frac{\hat{I}(\mathbf{x}) M(\mathbf{x})}{\hat{B}(\mathbf{x})}
$$

where $\hat{B}(\mathbf{x})$ is the local background value, calculated by substituting the argument $u$ in (4) with

$$
v=\left(\sqrt{(x-s)^{2}+(y-l)^{2}}-r_{2}-r_{3}\right) / r_{3} .
$$

By assuming the interactance values inside $A$ are independent and identical distributed, and using the model in (2), the variance for the local mean value is estimated by

$$
S_{\hat{I}}^{2}(\mathbf{x})=\frac{\hat{I}(\mathbf{x}) \sum_{(x, y) \in A} C(x) M(x, y) K^{2}(u)}{\sum_{(x, y) \in A} M(x, y) K^{2}(u)},
$$


where $C(x)$ is the calibration factor defined in (2). The variance for the local background, $S_{\hat{B}}^{2}(\mathbf{x})$, is calculated by substituting $u$ in 7 with $v$ from 6 .

\subsection{Detecting absorbing objects}

A pixel is defined as being on an absorbing object if its local mean value, $\hat{I}(\mathbf{x})$, is lower than its local background value, $\hat{B}(\mathbf{x})$. For a single band image the test operator is given as

$$
z_{S}(\mathbf{x})=\frac{\hat{I}(\mathbf{x})-\hat{B}(\mathbf{x})}{\sqrt{S_{\hat{I}}^{2}(\mathbf{x})+S_{\hat{B}}^{2}(\mathbf{x})}},
$$

Previous work by Stormo et al. (2007) has shown that the band ratios 458 $\mathrm{nm} / 752 \mathrm{~nm}$ and $517 \mathrm{~nm} / 752 \mathrm{~nm}$ enhance the contrast of pale and dark nematodes as compared to using any single band. Similar to (8) a pixel is defined as belonging to an absorbing object if the local mean value of the band ratio, $\hat{R}_{I}(\mathbf{x})$, has a lower value than the local reference value, $\hat{R}_{B}(\mathbf{x})$. The two mean values $\hat{R}_{I}(\mathbf{x})$ and $\hat{R}_{B}(\mathbf{x})$ are calculated from (4) by substituting the single plane image, $I(\mathbf{x})$, by the band ratio, $R(\mathbf{x})=I\left(\mathbf{x}, \lambda_{1}\right) / I\left(\mathbf{x}, \lambda_{2}\right)$. The test operator for the band ratio is defined as

$$
z_{R}(\mathbf{x})=\frac{\hat{R}_{I}(\mathbf{x})-\hat{R}_{B}(\mathbf{x})}{\sqrt{S_{\hat{R}_{I}}^{2}(\mathbf{x})+S_{\hat{R}_{B}}^{2}(\mathbf{x})}} .
$$

The variance for the local mean value is estimated by

$$
\begin{aligned}
S_{\hat{R}_{I}}^{2}(\mathbf{x}) & =\frac{\sum_{(x, y) \in A} K(u) M(x, y) \hat{I}(x, y)^{2}}{\sum_{(x, y) \in A} K(u) M(x, y)} \\
& -\left(\frac{\sum_{(x, y) \in A} K(u) M(x, y) \hat{I}(x, y)}{\sum_{(x, y) \in A} K(u) M(x, y)}\right)^{2},
\end{aligned}
$$


where $u$ is defined in 4 . The variance, $S_{\hat{R}_{B}}^{2}$, for the local reference value is calculated in a similar way, by substituting $u$ in (10) with $v$ from (6).

A pixel is defined as an absorbing object if the test operator in (8) or (9) is less then a threshold, $\alpha_{D}$

\subsection{Optimizing the preprocessing method}

The training spectra for the nematode class were selected from the center pixel of 100 nematodes, clearly visible in the hyperspectral images of the fillets in the training set $(\mathrm{N}=43)$. The images were all previously calibrated using the smoothed Teflon calibrated image, calculated by (4), and the local calibration filter calculated by (5). The calibrated spectra were then pretreated using each of the five methods explained in Rinnan et al. (2009): 1) Standard normal variate (SNV), 2) Multiplicative scatter correction (MSC), 3) Savitzky-Golay second derivative, 4) normalizing each spectrum with its Euclidean length and 5) normalizing each spectrum with the area under the spectrum curve.

The parameters for the five calibration methods were optimized over the equally spaced grid with parameters $r_{1}=\{0,0.5, \ldots, 3\}, r_{2}=\{1,1.5, \ldots, 5\}$, $r_{3}=\{1,1.5, \ldots, 5\}$ and $W_{S}=\{1,3,5\}$, where $r_{2} \geq r_{1}$ and $W_{s}$ is the width of the Savitzky-Golay filter. The parameters $r_{1}, r_{2}$ and $r_{3}$ are given in $\mathrm{mm}$, while $W_{S}$ is given in wavelength units of $10 \mathrm{~nm}$. In addition the amount of nematodes, defined as absorbing objects was varied in the range $D=$ $\{70 \%, 80 \%, \ldots, 100 \%\}$, and the corresponding threshold $\alpha_{D}$ was calculated using the manual labeled nematodes in the training set. Some pixels were defined as absorbing objects for all pre-treatment methods and parameters. The corresponding spectra were defined as the training samples from the 
other absorbing feature (OAF) class.

The Fisher transformation vector, w, was calculated as described in Duda et al. (2000, pg.120) using the spectra from the nematode class in the training set $(N=100)$ and equally many spectra, randomly selected, from the OAF class. The separation boundary for the fisher linear classifier is defined as $\mathbf{w}^{T} \mathbf{P}-h=0$, where $\mathbf{P}$ is the spectrum to be classified. By applying the Fisher linear classifier to the training set and varying the threshold, $h$, in the range corresponding to detection rate from $0-100 \%$ in $10^{5}$ equally spaced steps, the receiver operating characteristic curve (ROC) (Duda et al., 2000, pg.49) was calculated. This was repeated 100 times where new spectra representing the OAF class were extracted for each iteration. The mean and standard deviation for the area under the ROC curve were calculated, and used as a quantitative discriminant measure of each of the preprocessing methods and parameter sets.

\subsection{Automatic nematode detection}

A Gaussian maximum likelihood (GML) classifier (Duda et al., 2000) was used to classify pixels as nematodes or not based on their corresponding spectrum. Assuming identical prior probabilities for both classes, a pixel classified as a nematode will have a value of one if

$$
\log \left(L\left(R\left(\mathbf{x}_{i}\right) ; \mu_{n}, \Sigma_{n}\right)\right)-\log \left(L\left(R\left(\mathbf{x}_{i}\right) ; \mu_{b}, \Sigma_{b}\right)\right)>\beta
$$

where $R(\mathbf{x})$ is the local calibrated image, $\mu_{n}, \mu_{b}, \Sigma_{n}$ and $\Sigma_{b}$ is the maximum likelihood estimate of the mean and covariance for the nematode and background class respectively, $L(\cdot)$ is the multivariate normal likelihood function and $\beta$ is a threshold used for tuning the detection rate vs. the false alarm 
rate. The mean and covariance were estimated from the training set, using the center pixel from 100 nematodes for the nematode class and 100 absorbing pixels, randomly selected from each fillet, as the OAF class.

A new image, recorded by the spectrometer, is calibrated by (1) and the wavelength region truncated to $448-752 \mathrm{~nm}$. The image is further segmented using (3) and divided into its respective parts; Loin, belly and tail using the center line as a reference (Sivertsen et al., 2009). The image is then calibrated locally using (5), the spectra are pre-treated and each pixel classified by (11) as nematode or not. The result is region grown using dilation (Gonzalez et al., 2009) with a square 5x5 kernel of ones. If the region of connected pixels overlap with a manual labeled nematode, it is counted as a correct detection. If not, it is counted as a false alarm.

\section{Results}

\subsection{Optimal local calibration parameters}

By applying the Fisher linear classifier to the spectra in the training set, the ROC curve was calculated for all combinations of parameter sets and spectral pre-treatment methods. The area under the ROC curve for the best parameter sets, as a function of the pre-treatment method, was highest for SNV applied to the local calibrated image (Fig. 3A). The best parameter set for the local calibration filter was found using the band ratio $458 \mathrm{~nm} / 752 \mathrm{~nm}$ as a feature band and the parameters: $\left[r_{1}, r_{2}, r_{3}, \alpha_{D}\right]=[1,3,4,-7.65]$, and for the smoothed Teflon calibrated images pre-treated with SNV: $\left[r_{1}, \alpha_{D}\right]=$ $[1,-4.23]$.

The ROC curve for the SNV pre-treated spectra, from the training set, 
calibrated with the best parameters sets are shown in Fig. 3B. The training set contains only spectra from the center of hand picked nematodes clearly visible in the hyperspectral image. This was to reduce the risk of mixing the nematode spectra with the background muscle tissue. This is the main reason for the high performance of the Fisher linear classifier applied to the training set.

The local calibration filter rejected on average $89.57 \pm 1.29 \%$ of all pixels in the hyperspectral images from the training set due to the test in (9). The filter also has the effect of reducing the spatial variation for all bands (Fig. 4A and B), while the spectral features of the nematodes are enhanced (Fig. 4C). After the local calibration, four absorption peaks located approximately at 448, 547, 576 and $646 \mathrm{~nm}$ are visible (Fig. 4C). In addition, the effect of water absorption above $700 \mathrm{~nm}$ is no longer apparent (Fig. 4C). The peak observed at $430 \mathrm{~nm}$ in the Teflon calibrated spectra is shifted to approximately 448 $\mathrm{nm}$ after the local calibration. The two peaks, located at 547 and $576 \mathrm{~nm}$, are not visible for the embedded nematode (Fig. 4D).

\subsection{Industrial test}

The average length of the 43 fillets in the training set was measured by the segmentation software to $447.7 \pm 69.0 \mathrm{~mm}$, and 243 nematodes, all pale ones, were found by manually inspecting these fillets. No depth registration of the nematodes was done for the training set. For the fillets in the test set, the average length of the 127 fillets sampled before the trimming stations was measured to $546.2 \pm 81.7 \mathrm{~mm}$. In these fillets, 640 nematodes, $88.5 \%$ registered as pale and $11.5 \%$ registered as dark ones, were found by manual inspection. Of these, 13 pale and 3 dark nematodes were found lying 
detached on top of the fillet and were not located at the same position in the images. These nematodes were labelled UP (unknown position). The 20 fillets sampled after the trimming stations were measured to an average length of $542.4 \pm 90.4 \mathrm{~mm}$, and 18 small and pale nematodes were found in these fillets. The small and pale nematodes were most likely of the type $A$. Simplex, while the dark, and much larger, nematodes probably were P. Decipiens. The nematode distribution with respect to color, depth and position for all nematodes were calculated by the segmentation software. Most of the nematodes $(93 \%)$ were located in the Belly flap (Tab. 1). The local calibration method detected $81.5 \%$ of all nematodes as absorbing objects. Fewer of the pale nematodes were detected as absorbing objects with increasing depth. This was not observed for the dark nematodes.

No exact size measurement of the nematodes were done. However some of the smallest nematodes found were curled up in a circular shape with a diameter of approximately $1 \mathrm{~mm}$.

The nematode detection rate using the GML classifier was calculated as a function of fillets with one or more false alarms, sampled before and after the trimming stations (Fig. 5). By accepting that $60 \%$ of the fillets sampled before the trimming stations had one or more false alarms, as was done in Sivertsen et al. (2011a), the GML classifier achieved a detection rate of $52.4 \%$ for all nematodes (dark and pale ones), $50.7 \%$ for pale nematodes and $65.3 \%$ for the dark nematodes. Accepting the same false alarm rate in the fillets sampled after the trimming stations, the detection rate increased to $61.5 \%$ for all nematodes, $60.3 \%$ for pale nematodes and $70.8 \%$ for the dark nematodes. By extending the wavelength range from 440-752 nm to 
458-800 nm, as was used by Sivertsen et al. (2011a), no improvement was achieved (results not shown).

\section{Discussion}

Our results show that interactance hyperspectral imaging can be applied as a tool for automatic detection of nematodes in cod fillets, at the required industrial speed of $400 \mathrm{~mm} / \mathrm{second}$. Even though the false alarm rate is high, the system can reduce the workload for the trimmers significantly.

The proposed local calibration filter reduces intensity variations across the fillet area in the image and the spectral difference between nematodes and other absorbing objects is enhanced. The method reduces the number of pixels to classify by almost $90 \%$, while $81.6 \%$ of all nematodes are detected as absorbing objects. The nematode detection rate reported in this study is improved due to the local calibration method and, for pale nematodes, better then previously reported by Sivertsen et al. (2011a).

The two peaks observed at 540 and $576 \mathrm{~nm}$ in the local calibrated nematode spectra, being a signature of oxygenated haemoglobin $(\mathrm{OHb})$, is found in absorption spectra from white, red and brown nematodes (Heia et al., 2003; Dixon et al., 1993). In the present study the peak was located at 547 $\mathrm{nm}$ instead of $540 \mathrm{~nm}$, indicating a small calibration error due to the lower spectral resolution in the current spectrometer. The peak observed at 646 $\mathrm{nm}$, only observed in spectra from dark nematodes, is probably due to methaemoglobin $(\mathrm{MHb})$ having an absorption maximum at $632 \mathrm{~nm}$ (Olsen and Elvevoll, 2011). For nematodes embedded in the fish muscle, the two $\mathrm{OHb}$ peaks are not easily seen in the local calibrated spectra. Both fresh and 
frozen-thawed cod muscle show a clear absorption peak around $550 \mathrm{~nm}$ due to absorption of haemoglobin or myoglobin $(\mathrm{HHb})$ in the muscle (Sivertsen et al., 2011b). For an embedded nematode, where scattering of light in the cod muscle is prominent, the signature from the nematode is mixed with the signature from the surrounding muscle. Hence, the HHb peak from the muscle masks out the two $\mathrm{OHb}$ peaks found in the nematode spectra.

The detection rate reported from the test set, using the GML classifier, were higher for the dark nematodes than for the pale nematodes even though no dark nematodes were present in the training set. This indicate that similar mechanisms are attenuating light in these two nematode species. An explanation could be that there is another chromophore present in both pale and dark nematodes. From previous studies it is known that the connective tissue in nematodes contains elastin and collagen (Hafsteinsson and Rizvi, 1987). Elastin is a yellow insoluble protein, known to exhibit a brilliant ultraviolet induced fluorescence in the visible region (Thornhill, 1972). Pale nematodes are known to have a yellow color, and all nematodes exhibit a strong fluorescence in the visible region when illuminated at $360 \mathrm{~nm}$ (Pippy, 1970). It seems plausible that this is due to elastin. The implication of this is that also the dark nematodes, having a higher amount of haemoglobin, contains elastin and probably the reason why dark nematodes are detected so well by the GML classifier, even though only pale nematodes were used in training the classifier.

The reported manual detection rate under industrial conditions varies a great deal, and is reported in the range of 33 - $93 \%$ for heavy infected fillets and 70-100 \% for less infected fillets (Varga and Anderson, 1971). One of 
the largest studies on manual nematode detection performance, performed over one year, in three different factories and on 22000 fillets, reports an average detection rate of $68 \%$ (Bublitz and Choudhury, 1992). Both of these studies were performed in Canadian waters where A. Simplex rarely are found in the fillet; one study reporting a mean intensity of 0.038 A. Simplex per fillet (McClelland et al., 1983). In the Barents sea, outside northern Norway, A. Simplex is abundant were as much as $96 \%$ of the fillets have been reported infected, with a mean intensity of 6.1 nematodes per fillet (Aspholm, 1995). No reports have been found on manual detection rate for A. Simplex under industrial conditions, but the manual detection rate by destructively slicing the fillet, is reported to only $42 \%$ (McClelland et al., 1983), and as low as $7 \%$ when candling pelagic fish (Levsen et al., 2005). It is evident that the manual detection rate for A. Simplex is very low, also demonstrated in this study where 18 pale nematodes were found in the fillets sampled after the trimming stations. We therefore conclude that the previous studies on manual detection rate for nematodes under industrial conditions, in fact document the manual detection rate for $P$. decipiens. This corresponds well with the detection rate for the GML classifier applied to dark nematodes in the present study. For pale nematodes, the GML classifier performs better than what to be expected from manual inspection.

For fillets with skin on, the manual detection rate is reported to be only $25 \%$ (Hauksson, 1991). The system presented in this work has previously been used on both fillets with and without skin (Sivertsen et al., 2011b). The current salt fish production, where fillets are inspected with skin, would benefit from applying this system today. 
The detection rate for the GML classifier depends on the false alarm rate permitted in production. To compare the present study with that of Sivertsen et al. (2011a), we have specifically reported the detection rate for a false alarm rate of $60 \%$. The false alarm rate is reported as number of fillets with one or more false alarms. For operational use, the false alarm rate can be converted to false alarms per fillet area or fillet weight, making it invariant to variation in fillet size. The results show a clear difference in the number of false alarms detected in fillets sampled before and after the trimming stations. This is probably due to the fact that the trimmers removes some of the blood spots, having a similar spectral characteristic to nematodes. The nematodes found lying loose on top of the fillets might also have been counted as false alarms.

The factory, where the test was run, produced fresh loins for the European market. This is their high value product, and it needs to be shipped to the market as soon as possible. The inspection system presented here can be implemented in front of the trimming stations and in combination with a system for automatically portioning. The loin considered clean by the inspection system could then be sent directly to packing. This would mean a workload reduction for the trimmers and a better end product with a longer shelf life, due to a shorter time exposure to the high temperature of the trimming area. By applying the system after the trimming stations, as an extra control, we would expect more of the nematodes being detected but a significant workload increase on the trimming stations due to the high false alarm rate. An extra benefit with the system is the ability to classify the raw material based on freshness, or remaining shelf life, and on whether the raw material has been previously frozen or not (Sivertsen et al., 2011b). 
In this study the focus has been on the design of the inspection system and the local calibration method. In future studies we hope to improve the results by applying a more advanced classifier. In addition investigating how the current system applies to other species such as saith and haddock would be of great interest.

Aspholm, P., Jun. 1995. Anisakis simplex Rudolphi, 1809, infection in fillets of Barents Sea cod Gadus Morhua L. Fisheries Research 23 (3-4), 375-379.

Barnes, R., Dhanoa, M., Lister, S., 1989. Standard normal variate transformation and de-trending of near-infrared diffuse reflectance spectra. Applied Spectroscopy 43 (5), 772-777.

Barron, S. A., Flogstad, H. O., Blessinger, K. V., Oct. 1995. Digital black clamp.

Benvenuto, F., Camera, A. L., Theys, C., Ferrari, A., Lantéri, H., Bertero, M., Jun. 2008. The study of an iterative method for the reconstruction of images corrupted by Poisson and Gaussian noise. Inverse Problems 24 (3), 035016.

Bublitz, C., Choudhury, G., 1992. Effect of light intensity and color on worker productivity and parasite detection efficiency during candling of cod fillets. Journal of Aquatic Food Product Technology 1 (2), 75-89.

Dixon, B., Kimmins, W., Pohajdak, B., 1993. Variation in Colour of Pseudoterranova decipiens (Nematoda; Anisakidae) Larvae Correlates with Haemoglobin Concentration in the Pseudocoelomic Fluid. Canadian Journal of Fisheries and Aquatic Sciences 50 (4), 767-771. 
Duda, R. O., Hart, P. E., Stork, D. G., 2000. Pattern Classification (2nd Edition). Wiley-Interscience.

Epanechnikov, V. A., Jul. 1969. Non-Parametric Estimation of a Multivariate Probability Density. Theory of Probability and its Applications 14 (1), 153.

Fischler, C., 2002. Food selection and risk perception. In: Anderson, H., Blundell, J., Chiva, M. (Eds.), Food selection: from genes to culture. pp. $135-149$.

Folland, G., 1999. Real Analysis: Modern Techniques and Their Applications. John Wiley \& Sons.

Geladi, P., MacDougall, D., Martens, H., 1985. Linearization and scatter correction for Near-Infrared Reflectance Spectra of Meat. Applied Spectroscopy 39 (3), 491-500.

Gonzalez, R. C., Woods, R. E., Eddins, S. L., 2009. Digital Image Processing Using MATLAB, 2nd ed. Gatesmark Publishing.

Gowen, A., Odonnell, C., Cullen, P., Downey, G., Frias, J., Dec. 2007. Hyperspectral imaging - an emerging process analytical tool for food quality and safety control. Trends in Food Science and Technology 18 (12), 590-598.

Hafsteinsson, H., Parker, K., Chivers, R., Rizvi, S., 1989. Application of ultrasonic waves to detect sealworms in fish tissue. Journal of Food Science 54 (2), 244-247.

Hafsteinsson, H., Rizvi, S., 1987. A review of the sealworm problem. Journal of Food Protection 50 (1), 70-84. 
Hauksson, E., 1991. Parasitic nematodes in commercially important fish. In: Pau, L., Olafsson, R. (Eds.), Fish quality control by computer vision. New York:. Marcel Dekker, New York, pp. 77-93.

Heia, K., Nilsen, H., Sivertsen, A. H., 2003. Imaging Spectroscopy as a tool for detection of nematodes. Tech. rep., Fiskeriforskning, Troms $\varnothing$.

Heia, K., Sivertsen, A. H., Stormo, S. K., Elvevoll, E., Wold, J. P., Nilsen, H., Jan. 2007. Detection of nematodes in cod (Gadus morhua) fillets by imaging spectroscopy. Journal of food science 72 (1), E011-5.

Janesick, J. R., 2001. Scientific Charge-Coupled Devices. SPIE Publications.

Levsen, A., Lunestad, B. T., Berland, B., Apr. 2005. Low detection efficiency of candling as a commonly recommended inspection method for nematode larvae in the flesh of pelagic fish. Journal of food protection 68 (4), 828-32.

Marcogliese, D. J., Jan. 2002. Food webs and the transmission of parasites to marine fish. Parasitology 124, S83-99.

McClelland, G., Misra, R., Marcogliese, D., Fisheries, C. D., Oceans, Laboratory, O. H., 1983. Variations in abundance of larval anisakines, sealworm (Phocanema decipiens) and related species in cod and flatfish from the southern Gulf of St. Lawrence (4T) and the Breton Shelf (4Vn). Government of Canada, Fisheries and Oceans.

Olsen, S. H., Elvevoll, E. O., Feb. 2011. pH-induced shift in hemoglobin spectra: a spectrophotometeric comparison of atlantic cod ( Gadus morhua ) and mammalian hemoglobin. Journal of agricultural and food chemistry 59 (4), 1415-22. 
Ottestad, S., Hy, M., Stevik, A., Wold, J., 2009. Prediction of ice fraction and fat content in superchilled salmon by non-contact interactance near infrared imaging. Journal of Near Infrared Spectroscopy 17 (2), 77-87.

Petursson, J., 1991. Optical Spectra of Fish Flesh and Quality Defects in Fish. In: Pau, L., Olafsson, R. (Eds.), Fish Quality Control by Computer Vision. Marcel Dekker, New York, pp. 45-76.

Pippy, J. H. C., 1970. Use of ultraviolet light to find parasitic nematodes in situ. Journal of the Fisheries Research Board of Canada 27 (5), 963-965.

Rinnan, A. s., Berg, F. V. D., Engelsen, S. r. B., Nov. 2009. Review of the most common pre-processing techniques for near-infrared spectra. TrAC Trends in Analytical Chemistry 28 (10), 1201-1222.

Savitzky, A. P.-E. C., Golay, J. P.-E. C., 1964. Smoothing and differentiation of data by simplified least squares procedures. Analytical chemistry 36 (8), $1627-1639$.

Segtnan, V., Hø y, M., Lundby, F., Narum, B. r., Wold, J., 2009. Fat distribution analysis in salmon fillets using non-contact near infrared interactance imaging: a sampling and calibration strategy. Journal of Near Infrared Spectroscopy 17 (5), 247.

Sivertsen, A., Chu, C., Wang, L., Godtliebsen, F., Heia, K., Nilsen, H., Feb. 2009. Ridge detection with application to automatic fish fillet inspection. Journal of Food Engineering 90 (3), 317-324.

Sivertsen, A. H., Heia, K., Stormo, S. K., Elvevoll, E., Nilsen, H., Jan. 2011a. Automatic Nematode Detection in Cod Fillets (Gadus Morhua) by 
Transillumination Hyperspectral Imaging. Journal of Food Science 76 (1), S77-S83.

Sivertsen, A. H., Kimiya, T., Heia, K., Apr. 2011b. Automatic freshness assessment of cod (Gadus morhua) fillets by Vis/Nir spectroscopy. Journal of Food Engineering 103 (3), 317-323.

Snyder, D. L., Helstrom, C. W., Lanterman, A. D., Faisal, M., White, R. L., Feb. 1995. Compensation for readout noise in CCD images. Journal of the Optical Society of America A 12 (2), 272.

Stormo, S. K., Ernstsen, A., Nilsen, H., Heia, K., Sivertsen, A. H., Elvevoll, E., Jul. 2004. Compounds of parasitic roundworm absorbing in the visible region: target molecules for detection of roundworm in Atlantic cod. Journal of food protection 67 (7), 1522-5.

Stormo, S. K., Sivertsen, A. H., Heia, K., Nilsen, H., Elvevoll, E., Aug. 2007. Effects of single wavelength selection for Anisakid roundworm larvae detection through multispectral imaging. Journal of food protection 70 (8), 1890-1895.

Thornhill, D. P., Jan. 1972. Elastin: Locus and Characteristics of Chromophore and Fluorophore. Connective Tissue Research 1 (1), 21-30.

Varga, S., Anderson, N. E., 1971. Parasite infestation of cod fillets before and after candling in the Maritime area. Tech. rep., Applied Re- search and Development Laboratory, Canada Department of Fish, Forestry Maritime Area, Inspection Branch, Halifax, Nova Scotia, Canada. 
${ }_{528}$ Werner, M. T., Fæ ste, C. K., Levsen, A., Egaas, E., Oct. 2011. A quan529 titative sandwich ELISA for the detection of Anisakis simplex protein in 530 seafood. European Food Research and Technology 232 (1), 157-166.

531 Wharton, D. a., Aalders, O., Dec. 2002. The response of Anisakis larvae to 532 freezing. Journal of helminthology 76 (4), 363-8.

${ }_{533}$ Wold, J. P., Johansen, I.-R., Haugholt, H., Tschudi, J., Thielemann, J., 534 Segtnan, V. H., Wold, E., 2006. Non-contact transflectance near infrared 535 imaging for representative on-line sampling of dried salted coalfish (ba536 calao). Journal of Near Infrared Spectroscopy 14, 59-66. 




A



$\mathrm{B}$

Figure 1: A sketch showing the dimensions and position of the spectrometer and fiber lines (A) and a photo of the machine with the front cover removed (B). The light sources are connected to the two fiber lines through the black fiber cables seen in the photo. All measures are in $\mathrm{mm}$. 




Figure 2: The regions used for calculating the local mean value, $\hat{I}(\mathbf{x})$, and the local background value, $\hat{B}(\mathbf{x})$, at position, $\mathbf{x}=(s, l)$, for the highpass filter. 

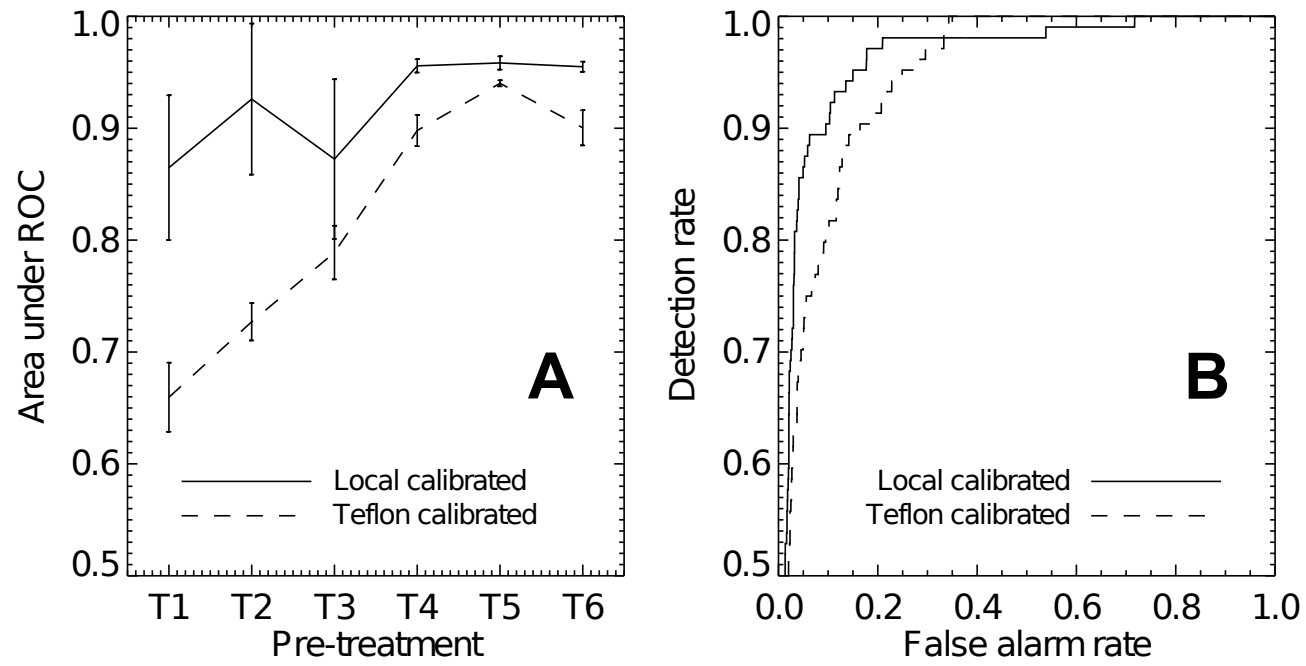

Figure 3: (A) The five calibration methods, with the highest average area under the ROC curve for the different pre-treatment methods: No spectral pre-treatment (T1), area normalization (T2), Euclidean length normalization (T3), MSC normalization (T4), SNV normalization (T5) and Savitzky-Golay second derivative with $W_{s}=3$ (T6). (B) The corresponding ROC curves for the best pre-treatment. 

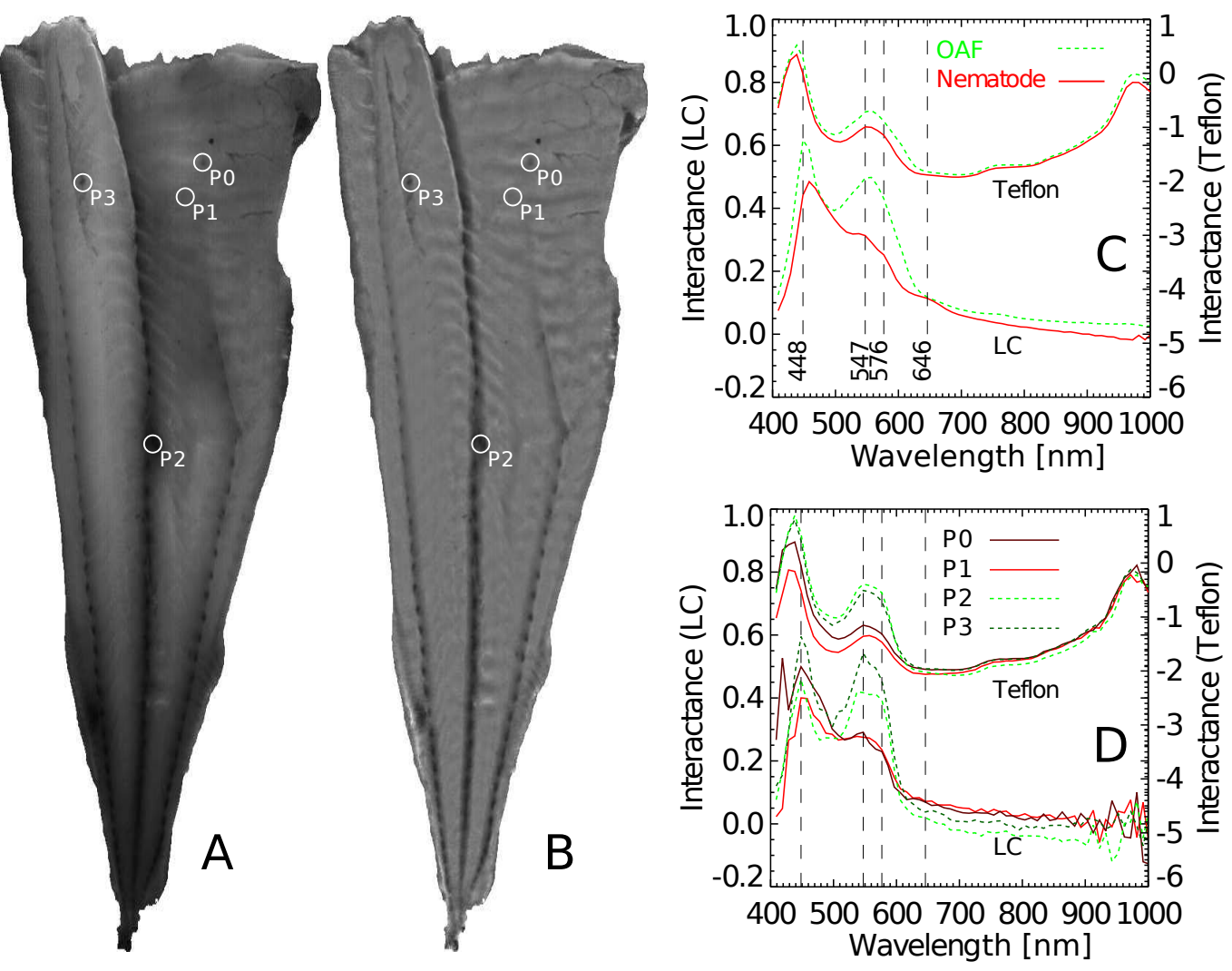

Figure 4: (A) The band ratio $458 \mathrm{~nm} / 752 \mathrm{~nm}$ for the Teflon calibrated image, and (B) the local calibrated image (LC). (C) The average nematode spectra and average spectra from other absorbing features (OAF) in the training set. (D) The spectra from the center pixels of a surface (P0) and embedded (P1) nematode, and two other absorbing features (P2 and P3). 


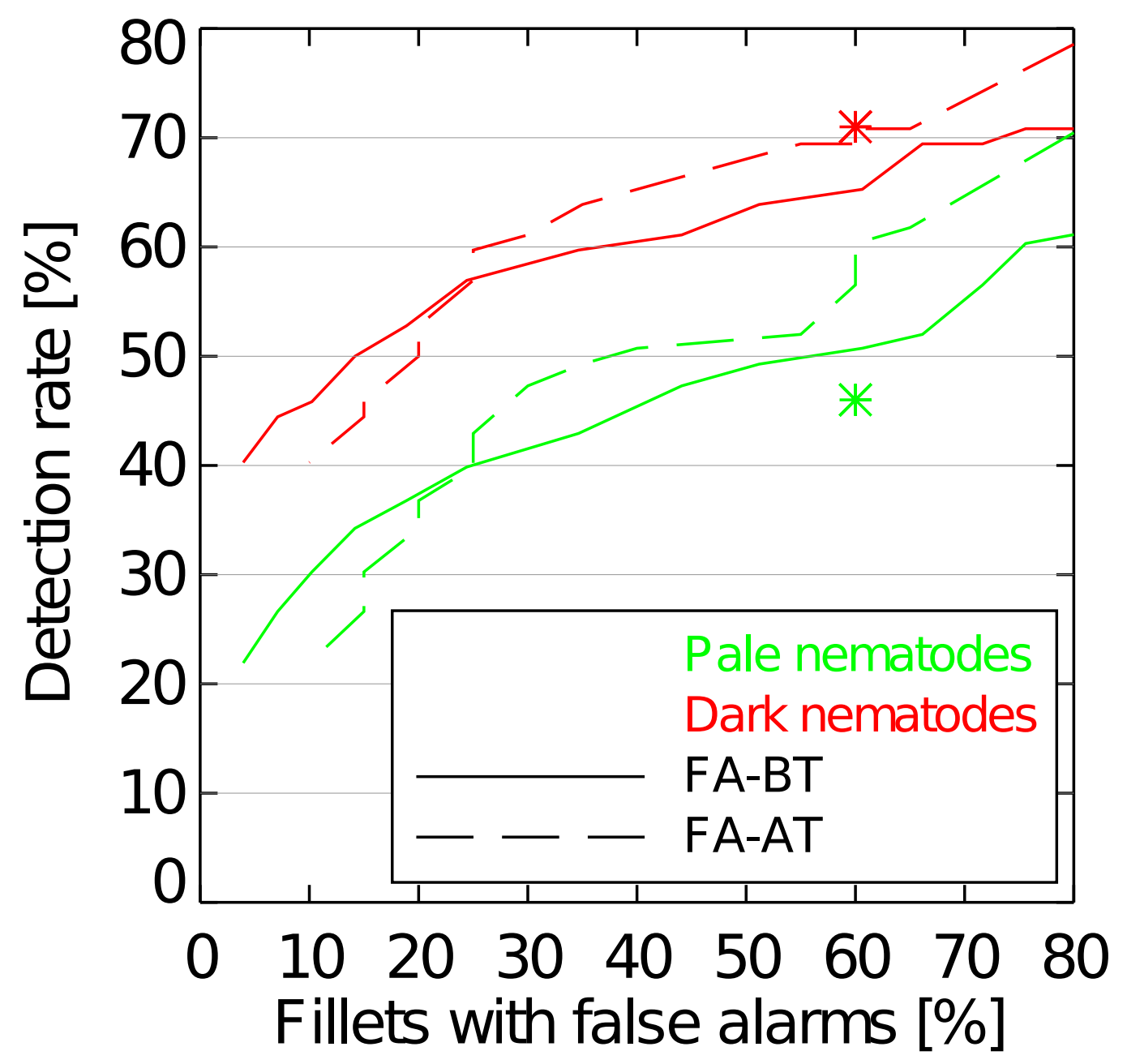

Figure 5: Performance of the GML classifier applied to all the fillets in the test set $(\mathrm{N}=147)$, as nematode detection rate vs. fillets with one or more false alarms. The false alarm is measured for the 127 fillets sampled before trimming (FA-BT) and the 20 fillets sampled after the trimming stations (FA-AT). The green and red symbols indicates the results reported by Sivertsen et al. (2011a) for pale and dark nematodes respectively. 


\begin{tabular}{|c|c|c|c|c|}
\hline & & $0-2 \mathrm{~mm}$ & $3-5 \mathrm{~mm}$ & $>6 \mathrm{~mm}$ \\
\hline \multirow{3}{*}{$\frac{0}{\widetilde{\sigma}}$} & Loin & $17(94.1 \%)$ & $13(76.9 \%)$ & $2(50.0 \%)$ \\
\hline & Belly & $249(88.0 \%)$ & $233(77.7 \%)$ & $37(43.2 \%)$ \\
\hline & Tail & $1(100 \%)$ & $0(-)$ & $0(-)$ \\
\hline \multirow{3}{*}{ 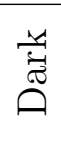 } & Loin & $0(-)$ & $4(100 \%)$ & $0(-)$ \\
\hline & Belly & $34(91.1 \%)$ & $28(92.9 \%)$ & $1(100 \%)$ \\
\hline & Tail & $4(75.0 \%)$ & $1(0 \%)$ & $0(-)$ \\
\hline
\end{tabular}

Table 1: Nematode distribution in the test set as a function of depth, color and position on the fillet. The number enclosed in brackets gives the amount of nematodes detected as absorbing objects by the local calibration method. 